\title{
NUEVOS REGISTROS DE HIEDEVIVOS \\ (INSECTA: HEMIPTERA: PENTATOMIDAE) PARA LA REPÚBLICA DOMINICANA Y LA HISPANIOLA, INCLUYENDO EL NUEVO HALLAZGO DE PIEZOSTERNUM SUBULATUM (THUNBERG, 1783)
}

\author{
Gabriel de los Santos ${ }^{1}$ y Ruth H. Bastardo ${ }^{2}$ \\ ${ }^{1}$ Museo Nacional de Historia Natural de Santo Domingo. Calle César Nicolás Penson, Plaza de la \\ Cultura, Santo Domingo, República Dominicana.g.delossantos@museohistorianatural.gov.do \\ ${ }^{2}$ Instituto de Investigaciones Botánicas y Zoológicas, Profesor Rafael M. Moscoso. Universidad \\ Autónoma de Santo Domingo, República Dominicana.r_bastardo@hotmail.com
}

\section{RESUMEN}

Se registran las especies Oebalus linki (Heidemann, 1917) y Podisus subferrugineus Barber et Bruner, 1932, por primera vez para la República Domincana y La Hispaniola. También se presenta el nuevo hallazgo de Piezosternum subulatum (Thunberg, 1783) luego de 200 años sin registros.

Palabras clave: Pentatomidae, nuevos registros, Antillas, Caribe.

Title: New records of stinkbugs (Insecta: Hemiptera: Pentatomidae) for the Dominican Republic and Hispaniola, including new collections of Piezosternum subulatum (Thunberg, 1783).

\section{ABSTRACT}

The species Oebalus linki (Heidemann, 1917) and Podisus subferrugineus Barber et Bruner, 1932, are the first time recorded for the Dominican Republic and Hispaniola. Also, the new finding of Piezosternum subulatum (Thunberg, 1783), after 200 years without record, is presented.

Key words: Pentatomidae, new records, Antilles, Caribbean.

Los insectos de la familia Pentatomidae (Hemiptera: Heteroptera) son conocidos con el nombre común de 'chinches' o 'chinches hediondas' ('stink bugs' en inglés) y en la República Dominicana como 'hiedevivos'. Esta familia posee reconocida importancia económica debido a que algunas de sus especies son consideradas plagas de cultivos. Por otra parte, algunos de los miembros de la subfamilia Asopinae presentan potencial como controles biológicos ya que son depredadores. A nivel mundial posee alrededor de 4,700 especies descritas, comprendidas en unos 900 géneros (Rider, 2006). Pérez-Gelabert y Thomas (2005) registran 75 especies para la República Dominicana y 77 para La Hispaniola (Haití y República Dominicana), de las cuales 14 son endémicas de la isla.

La fauna de pentatómidos de República Dominicana ha sido recientemente reevaluada (de los Santos, 2012), mediante el estudio de las colecciones del Museo Nacional de Historia Natural de Santo Domingo (MNHNSD) y el Instituto de Investigaciones Botánicas y Zoológicas (IIBZ) de la Universidad Autónoma de Santo Domingo. En dicho trabajo se encontraron dos especies que no habían sido registradas anteriormente para la República Dominicana y La Hispaniola: Oebalus linki (Heidemann), previamente solo conocida de Cuba e Isla de Pinos (Sailer, 1944; Alayo, 1967) y Podisus subferrugineus Barber et Bruner, anteriormente conocida 
de Cuba y Jamaica (Zayas, 1988; Thomas, 1992). Como bien indica Sailer (1944: 126), O. linki se distingue fácilmente de las demás especies del género por su pequeño tamaño y su corto escutelo. Esta especie no posee registros de daños a cultivos. En el caso de P. subferrugineus, esta se puede diferenciar de los demás Podisus Herrich-Schäffer, 1851, presentes en la isla por poseer el margen anterolateral del pronoto serrado, de color amarillento, y húmeros espinosos fuertemente pronunciados, proyectados horizontalmente. Se sabe que es una especie depredadora (al igual que los demás Podisus), pero no se tiene información sobre su dieta.

\section{NUEVO HALLAZGO DE PIEZOSTERNUM SUBULATUM (THUNBERG, 1783)}

En ambas colecciones se encontró a Piezosternum subulatum (Thunberg), especie colectada en las provincias de Monte Plata y Santiago (Fig. 1). Este constituye un nuevo hallazgo de esta especie en la República Dominicana y La Hispaniola desde Palisot de Beauvois a principios del siglo XIX, hace alrededor de 200 años (Pérez-Gelabert y Thomas, 2005: 347). Además de la República Dominicana, esta especie ha sido registrada para Puerto Rico y Cuba (Wolcott, 1948; Zayas, 1988). Sobre la escases de esta especie en las colecciones, Zayas (1988) comentó que nadie había conseguido un ejemplar en Cuba en los últimos 100 años, mientras que, por otro lado, Wolcott (1948) dice que en Puerto Rico se le puede encontrar en plantaciones de café o volando en El Yunque, y que también se encuentra en una variedad de hospederos a lo largo de la costa. Recientemente, Mateo y López (2010: 36) publicaron una fotografía de esta especie tomada en algún lugar de República Dominicana, aunque la especie fue erróneamente identificada como Nezara viridula (Linnaeus, 1758).

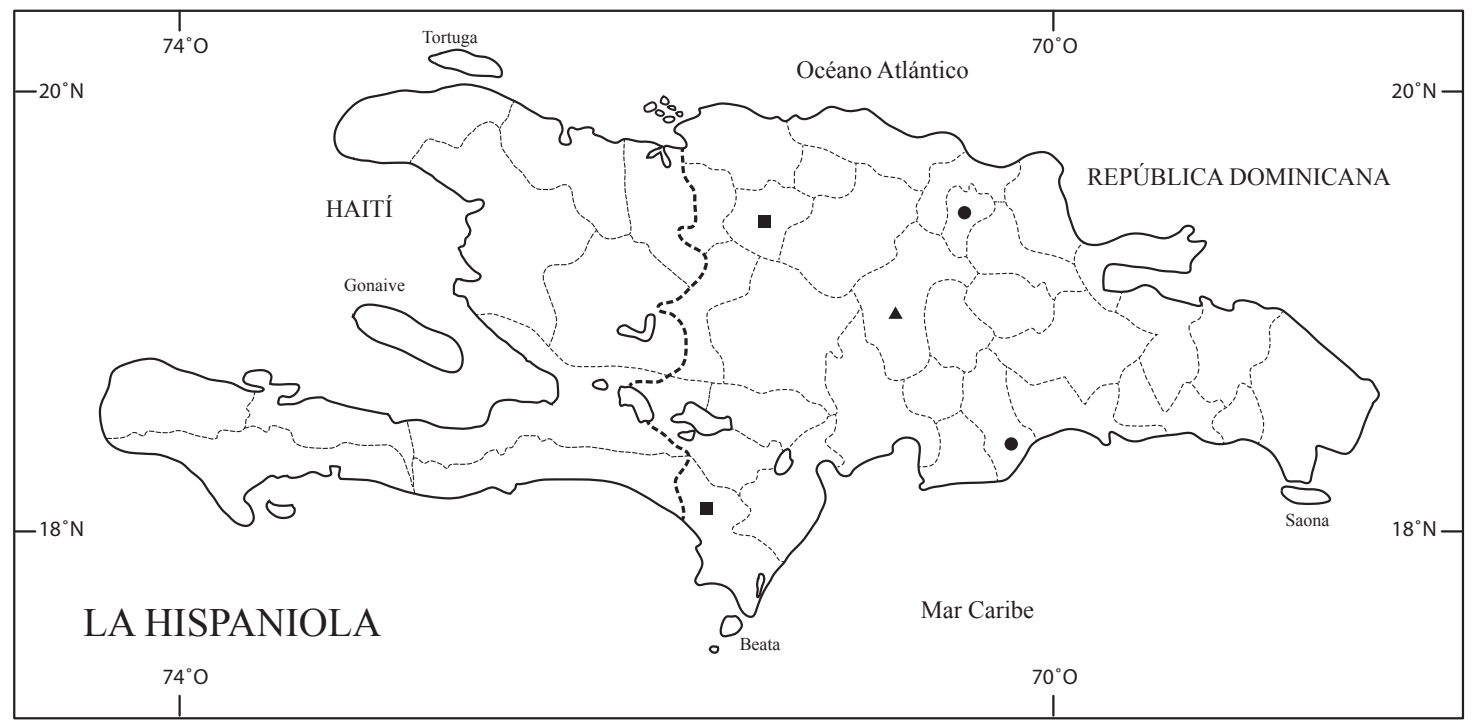

Figura 1. Localidades de colecta. Oebalus linki (Heidemann) (ם); Podisus subferrugineus Barber \& Bruner ( $\mathbf{\Delta})$; Piezosternum subulatum (Thunberg) (•).

\section{Material examinado:}

Subfamilia Asopinae

Podisus subferrugineus Barber \& Bruner, 1932

MNHNSD: 1 indet. MNHNSD 14.32, prov. La Vega, trail Los Tablones-La Ciénaga. 20.VI.2002, D. Pérez, B. Hierro, R. Bastardo. 


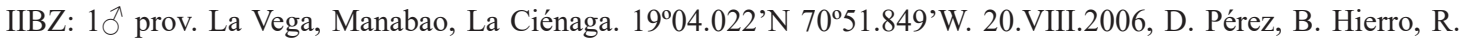
Bastardo.

Subfamilia Pentatominae

Oebalus linki (Heidemann, 1917)

MNHNSD: $1 \overbrace{}^{\Uparrow}$ MNHNSD 14.773, prov. Pedernales, Oviedo. 05-08.VI.2001, H. Takizawa.

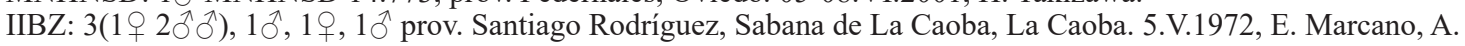
Abud \#11441, \#11485, \#11487, \#11453.

Subfamilia Tessaratominae

Piezosternum subulatum (Thunberg, 1783)

MNHNSD: $1 \widehat{\jmath}$ MNHNSD 14.1172, $1 \overbrace{}^{\Uparrow}$ MNHNSD 14.1173, prov. Hermanas Mirabal, Salcedo, Las Cuevas. 27. IV.1980, Reynoso. 1 NNNSD 14.1174, prov. Hermanas Mirabal, Salcedo, Las Cuevas. 03. IV.1980, Reynoso. IIBZ: $10 \hat{0}, 192$ inm. prov. San Cristóbal, Villa Altagracia, Mana de Haina, 6.II.1966, E. Marcano \#3103, \#3104.

\section{AGRADECIMIENTOS}

Al Dr. Donald B. Thomas (USDA-ARS, Subtropical Agriculture Research Center), por su valiosa y desinteresada ayuda. Al Dr. Brian Farrell (Museum of Comparative Zoology, Harvard University), por sus iniciativas en el desarrollo del convenio HARVARD-UASD, entre cuyos resultados se inscribe esta publicación. Al Dr. Daniel Pérez-Gelabert (U. S. National Museum of Natural History, Smithsonian Institution), por su ayuda con la literatura y todas sus sugerencias. A los directores Lic. Manuel Valdez (IIBZ) y MSc. Celeste Mir (MNHNSD) por las facilidades ofrecidas para la revisión de las colecciones. A Carlos Suriel, MNHNSD, por sus sugerencias.

\section{LITERATURA CITADA}

Alayo D., P. 1967. Catálogo de la fauna de Cuba - XVIII - Los Hemípteros de Cuba - II Familia Pentatomidae. Museo "Felipe Poey" de la Academia de Ciencias de Cuba, Trabajos de Divulgación No. 43, 1-47 + IX láminas.

De los Santos, G. 2012. Los Pentatómidos (Insecta: Hemiptera: Heteroptera: Pentatomidae) en las colecciones de referencia de la República Dominicana. Tesis de Licenciatura en Biología, Universidad Autónoma de Santo Domingo. V+105 pp.

Mateo, J. M. y A. López. 2010. Áreas protegidas de la República Dominicana, naturaleza en estado puro. Editora Amigo del Hogar, Santo Domingo. 767 pp.

Pérez-Gelabert, D. E., y D. B. Thomas. 2005. Stink bugs (Pentatomidae: Heteroptera) of the island of Hispaniola, with seven new species from the Dominican Republic. Boletín de la Sociedad Entomológica Aragonesa, 37: 319-352.

Rider, D. 2006. Number of Genera \& Species, Pentatomoidea Home Page. http://www.ndsu. nodak.edu/ndsu/rider/Pentatomoidea/Classification/Genus_Species_Numbers.htm. (02 de marzo, 2012).

Sailer, R. I. 1944. The Genus Solubea (Heteroptera: Pentatomidae). Proceedings of the Entomological Society of Washington. 46 (5): 105-127.

Thomas, D. B. 1992. Taxonomic synopsis of the Asopinae Pentatomidae (Heteroptera) of the Western Hemisphere. Thomas Say Monographs, Entomological Society of America, Lanham, Maryland, vol. 16: 1- 156. 
Wolcott, G. N. 1948. The insects of Puerto Rico. The Journal of Agriculture of the University of Puerto Rico, 32 (1): 1-224.

Zayas, F. de. 1988. Entomofauna Cubana: Tópicos entomológicos a nivel medio para uso didáctico. Tomo VII: Superorden Hemipteroidea, Orden Homoptera, Orden Heteroptera. Editorial Científico-Técnica, La Habana.

[Recibido: 13 de abril, 2012. Aceptado para publicación: 24 de mayo, 2012] 